\title{
Towards a Translational Approach to Food Addiction: Implications for Bulimia Nervosa
}

\author{
Monica Leslie $^{1} \cdot$ Ellen Lambert $^{1} \cdot$ Janet Treasure $^{1}$ \\ Published online: 25 June 2019 \\ (C) The Author(s) 2019
}

\begin{abstract}
Purpose of Review In recent years, the food addiction hypothesis of loss-of-control eating has gained traction in the field of eating disorders. In particular, the neural process of food addiction plays a dominant role in the recently formulated "addictive appetite" model of bulimia nervosa and binge eating disorder. Nonetheless, several components of the food addiction hypothesis, including the presence of withdrawal and tolerance effects, as well as the proposition that some foods possess "addicting" properties, remain highly controversial. In response, the current review synthesises existing evidence for withdrawal and tolerance effects in people with bulimia nervosa.

Recent Findings The recent development of a validated tool to measure withdrawal from highly processed foods will aid in measuring withdrawal symptoms and testing hypotheses related to withdrawal in the context of food addiction. We subsequently describe preclinical and human evidence for a central insulin- and dopamine-mediated pathway by which recurrent loss-ofcontrol binge eating is maintained in bulimia nervosa.

Summary Evidence in populations with bulimia nervosa and loss-of-control eating provides preliminary support for the role of food addiction in the maintenance of bulimia nervosa. Future longitudinal research is needed to develop a clearer profile of illness progression and to clarify the extent to which dysregulation in glucose metabolism contributes to food craving and symptom maintenance in bulimia nervosa.
\end{abstract}

Keywords Food addiction · Bulimia nervosa $\cdot$ Eating disorders $\cdot$ Sugar $\cdot$ Dopamine

\section{Background}

The concept of "food addiction" has received increasing attention in the scientific literature of recent years. While cogent arguments have been made against the establishment of food addiction as a psychiatric diagnosis in its own right [1], there is substantial evidence to suggest that processes similar to those observed in substance abuse disorders play a significant role in the maintenance of eating disorders in which loss of control of eating is a feature (e.g. anorexia nervosa-binge purge type, bulimia nervosa and binge eating disorder) [2•].

This article is part of the Topical Collection on Food Addiction

Monica Leslie monica.leslie@kcl.ac.uk

1 Institute of Psychiatry, Psychology and Neuroscience (IoPPN), Section of Eating Disorders, King's College London (KCL), 103 Denmark Hill, London SE5 8AF, UK
In this article, we will use bulimia nervosa (BN) as the exemplar. The "Addictive Appetite Model" proposes that three primary processes maintain psychopathology in $\mathrm{BN}$ : (1) the high salience of palatable foods [3], which is moderated by a genetic susceptibility to food approach tendencies, reduced efficiency in satiation processes [4] and/or episodes of food restriction; (2) chronic stress and interpersonal difficulties resulting in a deficiency of alternative rewards and a primed stress system [5]; and (3) large swings in blood glucose, caused by the consumption of foods with a high glycaemic index, self-induced vomiting or insulin resistance (and insulin omission in diabetes mellitus). These pathways may contribute to compulsive binge eating behaviour through aberrations in dopaminergic function in a similar way to substance addictions.

The current review presents a synthesis of the literature investigating some of the controversial aspects of applying the food addiction paradigm to eating disorders. For instance, there is uncertainty as to whether tolerance and withdrawal criteria for an addictive disorder as specified within the 
DSM-5 are met. We also present a synthesis of molecular, preclinical, clinical and neuroimaging evidence illustrating how fluxes in glucose and insulin moderate central dopaminergic functioning.

\section{Does Bulimia Nervosa Meet DSM-5 Criteria for an Addictive Disorder?}

The DSM-5 criteria for an addictive disorder are presented in Table 1. The extent to which BN meets these criteria has been reviewed extensively elsewhere [6]. In his 2014 review, Brewerton acknowledges significant phenotypic overlap between disorders of substance dependence and $\mathrm{BN}$ but highlights a paucity of systematic clinical evidence for tolerance and withdrawal in the latter, a point frequently cited as a major weakness of the food addiction hypothesis [7]. Much research on tolerance and withdrawal symptoms in humans to date has been largely anecdotal [8]. However, herein we synthesise the evidence from new assessment methods.

\section{Tolerance}

The most compelling evidence for food tolerance is demonstrated in animal models, as previously reviewed by Murray et al. [9]. Rats who voluntarily overeat highly palatable food exhibit evidence of a neural reward deficit due to downregulated dopamine D2 receptors, which worsens as weight is gained [10-12]. This decreased sensitivity to reward is directly linked to the onset of compulsive food seeking in rats [11]. Repetitive bingeing on sucrose interspersed with periods of dietary restriction causes rats to triple their overall daily sugar consumption [13], a finding which may be of particular importance to $\mathrm{BN}$ as it is characterised by intermittent fasting and binge episodes [14]. A similar downregulation of dopamine D2 receptors is found in humans addicted to drugs of abuse [15] and is thought to be a key driver of compensatory overconsumption in the Reward Hyposensitivity Theory [16, 17]. Behavioural observations in people with substance use disorders mirror these findings.

Individuals with $\mathrm{BN}$ endorse higher levels of tolerance-like symptoms measured using the Yale Food Addiction Scale (YFAS) [8], compared with healthy controls [18, 19]. There are clinical reports of subthreshold BN patients initiating larger and more frequent binge episodes over time [20]. Consistent with these accounts is cross-sectional evidence of the correlation between higher body weight and frequency and severity of binge eating episodes [21]. Individuals with bingetype eating disorders endorse significantly greater levels of eating for purposes of reward enhancement compared with weight-matched controls [22]. Such evidence, although compelling, remains indirect and is insufficient to prove the existence of tolerance in humans.

A preference for intensely sweet food and larger quantities of sweeteners in individuals with $\mathrm{BN}$ is a characteristic often presented as an indicator of tolerance [3, 23-25]. Furthermore, this preference remains after ingesting a glucose load [26]. Magnetic resonance imaging (MRI) studies indicate hypofunctioning of gustatory and limbic circuitry in BN patients when tasting palatable food compared with controls $[27,28]$ and compared with individuals recovered from $\mathrm{BN}[29,30]$. This evidence is consistent with the idea that individuals with BN ingest more food over time because of a decreased sensitivity to

Table 1 DSM-5 criteria for an addictive disorder (American Psychiatric Association, 2013)

\begin{tabular}{ll}
\hline Criteria 1 & The substance is often taken in larger amounts or over a longer period than was intended \\
Criteria 2 & There is a persistent desire or unsuccessful efforts to cut down or control use of the substance \\
Ariteria 3 & $\begin{array}{r}\text { A great deal of time is spent in activities necessary to obtain the substance, use the substance } \\
\text { or recover from its effects } \\
\text { Craving or a strong desire or urge to use the substance } \\
\text { Recurrent use of the substance despite having persistent or recurrent social or interpersonal problems caused } \\
\text { or exacerbated by the effects of its use } \\
\text { Criteria } 5\end{array}$ \\
Continued use of the substance despite having persistent or recurrent social or interpersonal problems caused \\
or exacerbated by the effects of its use
\end{tabular}

Bold text indicate criteria for an addictive disorder which are discussed in depth in the current manuscript with reference to their relevance to bulimia nervosa 
sweet taste resulting from repeated bingeing on hyperpalatable foods [27].

The development of impaired satiety mechanisms may be an indirect indicator of tolerance $[6,31,32]$. For example, a recent functional MRI (fMRI) study found that women in remission from BN exhibited the same response to taste stimuli in brain regions implicated in translating sensory information about taste into motivated behaviour, regardless of whether the individuals were hungry or sated, whilst healthy controls showed an increased response to taste stimuli when hungry versus when fed [33]. The authors also found an increased amygdala response in their remitted BN sample when fed compared to healthy controls, which they propose might project to the hypothalamus and motivate eating in the absence of hunger [33, 34]. It is possible that brain circuitry in BN fails to de-value food reward when in a fed state, leading to eating beyond metabolic need.

Five years on from Brewerton's 2014 review, there remains a paucity of direct evidence of tolerance in humans in relation to food intake and prospective, longitudinal studies are needed.

\section{Withdrawal Syndromes}

Preliminary evidence for a withdrawal syndrome in relationship to palatable food comes from animal models. There are consistent observations of strong physical (e.g. forepaw tremor, teeth chattering) and psychological (e.g. aggression, anxiety) withdrawal responses in rats during periods of withdrawal from sucrose [35-37]. The same observations, however, are not found with removal of high-fat foods [38] and have not yet been studied with removal of highly processed foods [39•]. Neuroimaging studies show patterns consistent with this behavioural data. Sugar-dependent rats show a significant increase in extracellular acetylcholine and a decrease in dopamine release in the nucleus accumbens shell, as compared to control groups, during a 36-h period of food deprivation [35], effects which are similar to withdrawal from morphine, nicotine and alcohol.

Traditionally, withdrawal symptoms have not been clearly defined in the context of addictive-like eating, prompting criticism of the food addiction framework [40]. To date, the food addiction field has largely relied on observational and anecdotal clinical reports based on small cohorts or single case studies [41-46] and on self-reported endorsement of withdrawal symptoms on the YFAS [18] and other withdrawal scales [47]. Cross-sectional self-report accounts are consistent in describing physiological symptoms of withdrawal similar to those experienced during opiate withdrawal $[8,48]$. Headaches, irritability and flu-like symptoms are reported by individuals abstaining from sugar [42], stomach pains, muscle spasms and shakiness by individuals abstaining or reducing intake of carbohydrates [43, 45] and nausea by individuals abstaining from salted food [49]. Furthermore, tiredness and irritability have been cited as motivating factors for eating [45], providing some suggestion of food being used as a "pick-me-up", or to avoid experiencing negative feelings stemming from withdrawal.

Symptoms of psychological withdrawal are also widely reported. Cross-sectional self-report accounts from patients with $\mathrm{BN}$ reveal that most feel tension, loneliness and physical symptoms of anxiety before a binge, and the majority feel that their negative psychological states are alleviated whilst engaged in a binge [39•]. Longitudinal studies using Ecological Momentary Assessment technology also report that binge eating and subsequent purging are usually preceded by dysphoric mood states [50-52]. However, there is high prevalence of depression and emotion dysregulation in $\mathrm{BN}$ populations [53], so it is not clear whether such presentations of low mood represent psychological withdrawal from food. Future research should aim to elucidate whether dysphoric mood states before bingeing are distinct from more permanent mood-related comorbidities, perhaps by comparing depressed versus non-depressed individuals with $\mathrm{BN}$.

The first and only tool to evaluate withdrawal in the context of addictive-like eating has been developed recently: the Highly Processed Food Withdrawal Scale-ProWS [39•]. This in part is derived from the premise that specific nutritional ingredients are capable of triggering addictive-like responses [54]. In a pilot study, the ProWS was found to be positively associated with elevated YFAS symptoms, BMI and weight cycling in a community sample, and responses on the ProWS explained an additional $11.2 \%$ of the variance in self-reported dieting success [39॰]. This tool may help differentiate between the withdrawal effects from different types of palatable food $[55,56]$.

\section{The Impact of Glucose Metabolism on Hedonic Eating Behaviour}

\section{Preclinical Evidence}

Sweet, pa latable foods act as unconditioned rewarding stimuli in humans and rodent models, with evidence suggesting that merely tasting sucrose without digestion produces activation of dopaminergic circuits within the striatum [57]. However, there is evidence to suggest that palatable foods with a high glycaemic index further contribute to the development of compulsive binge eating behaviour through changes in dopaminergic functioning triggered by wide swings in blood glucose. One candidate mechanism for this effect relates to the interaction between insulin and dopaminergic functioning.

The role of mesolimbic dopaminergic functioning in food approach behaviours has been reviewed extensively elsewhere [58]. Dopamine-deficient mouse models exhibit severe 
aphagia leading to weight loss and death [59]. Conversely, the stimulation of dopaminergic activity within the striatum triggers food consumption in rats without enhancing "liking" responses (e.g. lip-licking and paw licking). Thus, dopaminergic functioning is thought to hold a role in food approach behaviours (wanting) that is discrete from the hedonic response to food receipt [60]. In contrast, central insulin suppresses feeding [61]. It is thought that the effects of central insulin and dopamine on food intake are not independent but rather interact to regulate hedonic eating behaviour.

Dopaminergic neurons within the ventral tegmental area (VTA) express insulin receptors [62, 63], presenting a possible mechanism by which insulin might influence the dopaminergic induction of feeding behaviour. Furthermore, central insulin enhances the expression of dopamine transporter protein within the VTA via a protein kinase B (Akt) signalling system $[64,65]$. The enhanced expression of dopamine transporters on the cell surface induced by insulin exposure is associated with greater dopamine uptake [66], thus reducing levels of synaptic dopamine.

With regard to the effects of insulin on postsynaptic dopaminergic signalling, in vitro studies have found that insulin exposure invokes long-term depression of excitatory signalling within VTA dopamine neurons extracted from male C57BL/6J mice [67]. This effect appears to be long-lasting as, once induced, the long-term depression of VTA dopamine cells is not reversed by application of the insulin receptor antagonist S961 or through a tyrosine kinase inhibitor, which suppresses insulin receptor functioning [67].

The effects of central insulin on dopaminergic functioning within the VTA likely have downstream effects in suppressing feeding and particularly hedonic feeding. For example, Bruijnzeel et al. [68] found that injecting insulin directly into the VTA of female rats decreased 24-h food intake. Mebel et al. [69] have similarly found that injecting insulin directly into the VTA suppresses subsequent feeding in male C57BL/ $6 \mathrm{~J}$ mice; however, this effect was dependent on the hunger status of the animals. That is, insulin in the VTA suppressed the quantity of sweetened high-fat food consumed by sated mice but did not affect normal chow intake in hungry mice. This pattern of effects therefore suggests that insulin activity in the VTA acts selectively to suppress subsequent hedonic feeding, with weaker evidence for effects on homeostatic feeding behaviour.

Central insulin functioning may play a role in blocking the memory of palatable food reward or attenuating the incentive salience of cues associated with palatable food. Evidence for this hypothesis comes from studies demonstrating that injecting insulin either into the cerebral ventricles [70] or VTA [67] of rats at the time of memory retrieval reduces conditioned place preference for palatable food. Furthermore, Bruijnzeel et al. [68] found that injecting insulin at a dose of $0.005 \mathrm{mU} /$ side into the VTA elevated the reward threshold for intracranial self-stimulation, thus indicating a reduction of reward functioning.

\section{Evidence in Humans}

Insulin resistance impacts on central dopaminergic systems in humans. For example, Dunn et al. [71] conducted a positron emission tomography (PET) study using the dopamine D2/D3 receptor radioligand $[18 \mathrm{~F}]$ fallypride and found that insulin sensitivity is negatively correlated with dopamine type 2 receptor availability in the ventral striatum in a heterogeneous sample of lean and obese women. In men, Anthony et al. [72] found that exogenously administered insulin increases metabolism in the ventral striatum and prefrontal cortex, whilst decreasing metabolism in the right amygdala, hippocampus and cerebellar vermis. Furthermore, the effect of insulin in increasing metabolism in the ventral striatum and prefrontal cortex was lower in insulin-resistant versus insulin-sensitive participants [72]. This pattern of findings is thus indicative of traitlevel differences in the effects of central insulin on dopaminergic mesolimbic regions, known to be critical for food craving and food approach behaviour [59, 73].

Interactions between insulin resistance and central dopaminergic functioning may have functional significance for food craving in humans. Chechlacz et al. [74], in an fMRI study, found that people with type II diabetes mellitus (characterised by insulin resistance) exhibit greater blood oxygenated level dependent (BOLD) response to food versus non-food images in the insula, orbitofrontal cortex (OFC) and basal ganglia, when compared to people without diabetes mellitus. Moreover, this increased activation within the insula and OFC is positively correlated with self-reported external eating. These findings, taken together, thus provide evidence that insulin resistance, commonly observed following repeated excess consumption of fructose in combination with an overall excessive energy intake [75], is positively correlated with a pattern of neural response to food stimuli which is associated with greater external cue-driven eating. However, it should be noted that the correlational nature of these findings limits the ability to draw firm causal inferences. Although there is relatively less evidence regarding food craving in type I diabetes, an fMRI study has found that insulin detemir, which more readily enters the brain compared to standard forms of insulin, is associated with reduced BOLD response to food images in the bilateral insula, a brain region associated with the regulation of appetite [76]. The authors have speculated that insulin detemir may therefore induce a more effective satiety reaction, thus explaining the reduced levels of weight gain observed in people with type I diabetes mellitus taking insulin detemir [77].

In a related study, Jastreboff et al. [78] recruited 25 men and women in the obese weight range and 25 lean controls. 
Fasting insulin and glucose were taken to measure insulin resistance. During a subsequent fMRI task, audio scripts designed to provoke relaxing imagery or favourite food imagery were played. Food craving was assessed before and after each imagery trial. The degree of food craving following food imagery trials was positively associated with insulin resistance in the obese, but not lean, participant group. Furthermore, the relationship between insulin resistance and food craving within the obese participant group was mediated by BOLD responses in dopaminergic regions including the VTA and substantia nigra. These studies suggest that insulin resistance moderates craving and associated neural circuits in response to food-related imagery.

Thus, the above studies illustrate that interactions between central insulin and dopaminergic systems, known to impact on feeding behaviour in animals, also regulate food craving in humans. Whilst this evidence therefore supports a potential mechanism linking the short- and long-term physiological effects of sugar consumption to food craving in humans, it is also of interest to disentangle the effects of sweet taste on dopaminergic incentive sensitisation from the physiological effects of sugar in food approach behaviour in humans. In support of the physiological effects of glucose consumption on central dopaminergic functioning, regardless of sweet taste, Haltia et al [79] found that the intravenous administration of glucose, versus placebo, was associated with increased D2 receptor binding potential in the right caudate nucleus and bilateral putamen in both lean and overweight women. However, intravenous glucose administration was rather associated with reductions in $\mathrm{D} 2$ receptor binding potential in the bilateral caudate nucleus, left putamen and right thalamus in men. It should be noted that the intravenous method of administration employed in this study bypassed the gastrointestinal system, thus failing to stimulate the production of gastrointestinal hormones, such as glucagon-like peptide 1, which also impact on appetitive functioning [80,81]. This study is therefore limited in the extent to which it directly bears upon the oral consumption of glucose versus caloriefree sweet taste. Nonetheless, these findings provide evidence for the impact of glucose on mesolimbic dopaminergic functioning in the absence of sweet taste. The functional significance of the sexual dimorphism in brain response is not yet clear and would be an interesting avenue for future research.

In another PET study conducted in 19 participants with BMIs ranging from the lean to obese weight range, dopamine functioning was measured following the consumption of a 75 -g oral glucose drink versus a calorie-free sucralose drink of equal volume and sweetness. Within the lean participant group, consuming the glucose drink, versus the calorie-free sucralose drink, was associated with increased dopaminergic binding potential within the ventral striatum, whilst the opposite was observed in the obese participant group [82]. Thus, there is evidence that glucose impacts upon dopaminergic functioning separately from the effects of sweet taste alone, with BMI modulating the direction of that effect. The reduced activation stimulated by sugar consumption in obese participants is in line with previous evidence of downregulated striatal response to the receipt of sugar solutions, including chocolate milk and milkshakes [83, 84].

There is a relative paucity of research investigating the effects of glucose metabolism on dopamine-mediated feeding in $\mathrm{BN}$ and binge eating disorder without obesity. A recent meta-analysis of studies analysing insulin sensitivity in $\mathrm{BN}$ and binge eating disorders has found significantly reduced insulin sensitivity in both disorders [85••]. Such insulin resistance therefore leads to greater flux in blood glucose following the consumption of foods with a high glycaemic index, thus potentially contributing to food craving in a similar manner to that described above for populations with obesity [78]. The effect of insulin resistance on glucose flux is further exacerbated by the wide swings in blood glucose induced by intermittent fasting followed by objectively large binge eating episodes in people with BN [86]. Frank et al. [87] found evidence of some trait differences in brain response to glucose, with participants recovered from BN exhibiting suppressed BOLD response to a glucose, versus artificial saliva solution, in the anterior cingulate cortex and left cuneus in comparison to the control group. However, this study is confounded by the difference in sweet taste as well as nutritional content (glucose versus calorie-free liquid). It will therefore be critical to carry out similar research to that described above for obesity in populations with bulimia-spectrum disorders in order to clarify the functional role of a glucose metabolism pathway in lossof-control binge eating versus the chronic overeating which commonly characterises overweight and obesity.

\section{Conclusion}

The current literature review has thus far served to illustrate the existing state of the evidence with regard to food tolerance and withdrawal effects in BN. Additionally, preclinical and preliminary evidence in human studies has elucidated an insulin-dependent mechanism whereby foods with a high glycaemic index interact with mesolimbic dopamine systems and heighten food craving in cases of insulin dysregulation. Nonetheless, there are several lines of evidence that should be explored further before definite conclusions can be drawn with regard to withdrawal and tolerance in $\mathrm{BN}$ and the physiological mechanisms which maintain addictive responses to palatable food. 
Funding ML was supported by grants from the Swiss Fund for Anorexia Nervosa. JT was supported by the National Institute for Health Research (NIHR) Mental Health Biomedical Research Centre at South London and Maudsley NHS Foundation Trust and King's College London.

\section{Compliance with Ethical Standards}

Conflict of Interest Ms Leslie reports grants from the Swiss Fund for Anorexia Nervosa, from null, during the conduct of the study; other from Opiant Pharmaceuticals Inc., outside the submitted work. Dr. Treasure has nothing to disclose. Ms Lambert has nothing to disclose.

Human and Animal Rights and Informed Consent This article does not contain any studies with human or animal subjects performed by any of the authors.

Open Access This article is distributed under the terms of the Creative Commons Attribution 4.0 International License (http:// creativecommons.org/licenses/by/4.0/), which permits unrestricted use, distribution, and reproduction in any medium, provided you give appropriate credit to the original author(s) and the source, provide a link to the Creative Commons license, and indicate if changes were made.

\section{References}

Papers of particular interest, published recently, have been highlighted as:

- Of importance

•- Of major importance

1. Finlayson G. Food addiction and obesity: unnecessary medicalization of hedonic overeating. Nature Reviews Endocrinology. 2017;13(8):493.

2. Treasure J, Leslie M, Chami R, Fernández-Aranda F. Are trans diagnostic models of eating disorders fit for purpose? A consideration of the evidence for food addiction. European Eating Disorders Review. 2018;26(2):83-91 This paper presents a new maintenance model of recurrent loss-of-control binge eating: the addictive appetite model of bulimia and binge eating disorder. This paper posits the existence of specific maintenance processes related to food addiction, which can be formulated as falsifiable hypotheses.

3. Berridge $\mathrm{KC}$, Robinson TE. What is the role of dopamine in reward: hedonic impact, reward learning, or incentive salience? Brain research reviews. 1998;28(3):309-69.

4. Carnell S, Wardle J. Appetite and adiposity in children: evidence for a behavioral susceptibility theory of obesity. The American journal of clinical nutrition. 2008;88(1):22-9.

5. Turton R, Chami R, Treasure J. Emotional eating, binge eating and animal models of binge-type eating disorders. Current obesity reports. 2017;6(2):217-28.

6. Brewerton TD. Are eating disorders addictions? In: Eating disorders, addictions and substance use disorders. Berlin: Springer; 2014. p. 267-99.

7. Ziauddeen H, Farooqi IS, Fletcher PC. Obesity and the brain: how convincing is the addiction model? Nature Reviews Neuroscience. 2012;13(4):279.

8. Gearhardt AN, Corbin WR, Brownell KD. Preliminary validation of the Yale food addiction scale. Appetite. 2009;52(2):430-6.
9. Murray S, Gordillo M, Avena NM. Animal models of eating disorders, substance use disorders, and addictions. In: Eating disorders, addictions and substance use disorders. Berlin: Springer; 2014. p. 3-21.

10. Colantuoni $\mathrm{C}$, et al. Excessive sugar intake alters binding to dopamine and mu-opioid receptors in the brain. Neuroreport. 2001;12(16):3549-52.

11. Johnson PM, Kenny PJ. Dopamine D2 receptors in addiction-like reward dysfunction and compulsive eating in obese rats. Nature neuroscience. 2010;13(5):635.

12. Robinson MJ, et al. Individual differences in cue-induced motivation and striatal systems in rats susceptible to diet-induced obesity. Neuropsychopharmacology. 2015;40(9):2113.

13. Rada P, avena N, Hoebel B. Daily bingeing on sugar repeatedly releases dopamine in the accumbens shell. Neuroscience. 2005; 134(3):737-44.

14. American Psychiatric Association. Diagnostic and statistical manual of mental disorders. Arlington: American Psychiatric Publishing; 2013.

15. Koob GF, Volkow ND. Neurobiology of addiction: a neurocircuitry analysis. The Lancet Psychiatry. 2016;3(8):760-73.

16. Blum K, et al. The reward deficiency syndrome: a biogenetic model for the diagnosis and treatment of impulsive, addictive and compulsive behaviors. Journal of psychoactive drugs. 2000;32(sup1):1112.

17. Rogers PJ. Food and drug addictions: similarities and differences. Pharmacology Biochemistry and Behavior. 2017;153:182-90.

18. Meule A, Gearhardt AN. Five years of the Yale Food Addiction Scale: taking stock and moving forward. Current Addiction Reports. 2014;1(3):193-205.

19. de Vries SK, Meule A. Food addiction and bulimia nervosa: new data based on the Yale Food Addiction Scale 2.0. European Eating Disorders Review. 2016;24(6):518-22.

20. Stice E, Marti CN, Shaw H, Jaconis M. An 8-year longitudinal study of the natural history of threshold, subthreshold, and partial eating disorders from a community sample of adolescents. Journal of abnormal psychology. 2009;118(3):587.

21. Picot AK, Lilenfeld LR. The relationship among binge severity, personality psychopathology, and body mass index. International Journal of eating disorders. 2003;34(1):98-107.

22. Leslie M, Turton R, Burgess E, Nazar BP, Treasure J. Testing the addictive appetite model of binge eating: The importance of craving, coping, and reward enhancement. Eur Eat Disord Rev. 2018.

23. Franko DL, Wolfe BE, Jimerson DC. Elevated sweet taste pleasantness ratings in bulimia nervosa. Physiology \& behavior. 1994;56(5):969-73.

24. Drewnowski A, Bellisle F, Aimez P, Remy B. Taste and bulimia. Physiology \& behavior. 1987;41(6):621-6.

25. Drewnowski A, Shrager EE, Lipsky C, Stellar E, Greenwood M. Sugar and fat: sensory and hedonic evaluation of liquid and solid foods. Physiology \& behavior. 1989;45(1):177-83.

26. Rodin J, Bartoshuk L, Peterson C, Schank D. Bulimia and taste: possible interactions. Journal of Abnormal Psychology. 1990;99(1):32.

27. Bohon C, Stice E. Reward abnormalities among women with full and subthreshold bulimia nervosa: a functional magnetic resonance imaging study (in English). International Journal of Eating Disorders. 2011;44(7):585-95.

28. Frank GK, Reynolds JR, Shott ME, O'Reilly RC. Altered temporal difference learning in bulimia nervosa. Biological psychiatry. 2011;70(8):728-35.

29. Oberndorfer TA, et al. Altered insula response to sweet taste processing after recovery from anorexia and bulimia nervosa. Am J Psychiatry. 2013;170(10):1143-51.

30. Radeloff D, et al. High-fat taste challenge reveals altered striatal response in women recovered from bulimia nervosa: a pilot study. World J Biol Psychiatry. 2014;15(4):307-16. 
31. Haedt-Matt AA, Keel PK. Revisiting the affect regulation model of binge eating: a meta-analysis of studies using ecological momentary assessment. Psychological bulletin. 2011;137(4):660.

32. Halmi KA, Sunday S, Puglisi A, Marchi P. Hunger and satiety in anorexia and bulimia nervosa. Annals of the New York Academy of Sciences. 1989;575(1):431-45.

33. Ely AV, et al. Response in taste circuitry is not modulated by hunger and satiety in women remitted from bulimia nervosa. Journal of abnormal psychology. 2017;126(5):519.

34. Sun X, et al. Basolateral amygdala response to food cues in the absence of hunger is associated with weight gain susceptibility. Journal of Neuroscience. 2015;35(20):7964-76.

35. Avena NM, Rada P, Hoebel BG. Evidence for sugar addiction: behavioral and neurochemical effects of intermittent, excessive sugar intake. Neuroscience \& Biobehavioral Reviews. 2008;32(1):2039.

36. Iemolo A, et al. Withdrawal from chronic, intermittent access to a highly palatable food induces depressive-like behavior in compulsive eating rats. Behavioural pharmacology. 2012;23:593.

37. Wideman C, Nadzam G, Murphy H. Implications of an animal model of sugar addiction, withdrawal and relapse for human health. Nutritional neuroscience. 2005;8(5-6):269-76.

38. Bocarsly ME, Berner LA, Hoebel BG, Avena NM. Rats that binge eat fat-rich food do not show somatic signs or anxiety associated with opiate-like withdrawal: implications for nutrient-specific food addiction behaviors. Physiology \& behavior. 2011;104(5):865-72.

39. Schulte EM, Smeal JK, Lewis J, Gearhardt AN. Development of the highly processed food withdrawal scale. Appetite. 2018;131: 148-54 This paper describes the development and validation of the Highly Processed Food Withdrawal Scale (ProWS), which is the first validated psychometric scale to measure withdrawal symptoms in the context of food addiction. This paper presents data supporting the convergent validity of the ProWS with addictive-like eating and the predictive validity of the ProWS for dieting success.

40. Ziauddeen H, Fletcher PC. Is food addiction a valid and useful concept? obesity reviews. 2013;14(1):19-28.

41. Hansen S. Conceptualizing bulimia as addiction: a resident's personal experience. American Journal of Psychiatry Residents' Journal. 2016;11(08):6-8.

42. Davis C, Carter JC. Compulsive overeating as an addiction disorder. A review of theory and evidence. Appetite. 2009;53(1):1-8.

43. Thornley S, McRobbie H. Carbohydrate withdrawal: is recognition the first step to recovery? The New Zealand Medical Journal (Online). 2009;122(1290).

44. Gearhardt AN, Corbin WR, Brownell KD. Development of the Yale Food Addiction Scale Version 2.0. Psychology of Addictive Behaviors. 2016;30(1):113.

45. Ifland J, et al. Refined food addiction: a classic substance use disorder. Medical hypotheses. 2009;72(5):518-26.

46. Lingswiler VM, Crowther JH, Stephens M. Affective and cognitive antecedents to eating episodes in bulimia and binge eating. International Journal of Eating Disorders. 1989;8(5):533-9.

47. Gilbert DG, Gilbert BO, Schultz VL. Withdrawal symptoms: individual differences and similarities across addictive behaviors. Personality and individual Differences. 1998;24(3):351-6.

48. Farrell M. Opiate withdrawal. Addiction. 1994;89(11):1471-5.

49. Tekol Y. Salt addiction: a different kind of drug addiction. Medical hypotheses. 2006;67(5):1233-4.

50. Anestis MD, Selby EA, Crosby RD, Wonderlich SA, Engel SG, Joiner TE. A comparison of retrospective self-report versus ecological momentary assessment measures of affective lability in the examination of its relationship with bulimic symptomatology. Behaviour Research and Therapy. 2010;48(7):607-13.

51. Smyth JM, et al. Daily and momentary mood and stress are associated with binge eating and vomiting in bulimia nervosa patients in the natural environment. Journal of consulting and clinical psychology. 2007;75(4):629.

52. Selby EA, et al. Momentary emotion surrounding bulimic behaviors in women with bulimia nervosa and borderline personality disorder. Journal of Psychiatric Research. 2012;46(11):1492-500.

53. Braun DL, Sunday S, Halmi K. Psychiatric comorbidity in patients with eating disorders. Psychological medicine. 1994;24(4):859-67.

54. Pursey KM, Davis C, Burrows TL. Nutritional aspects of food addiction. Current Addiction Reports. 2017;4(2):142-50.

55. Curtis C, Davis C. A qualitative study of binge eating and obesity from an addiction perspective. Eating disorders. 2014;22(1):19-32.

56. Schulte EM, Avena NM, Gearhardt AN. Which foods may be addictive? The roles of processing, fat content, and glycemic load. PloS one. 2015;10(2):e0117959.

57. Avena NM, Rada P, Moise N, Hoebel B. Sucrose sham feeding on a binge schedule releases accumbens dopamine repeatedly and eliminates the acetylcholine satiety response. Neuroscience. 2006;139(3):813-20.

58. Berridge KC, Ho C-Y, Richard JM, DiFeliceantonio AG. The tempted brain eats: pleasure and desire circuits in obesity and eating disorders. Brain research. 2010;1350:43-64.

59. Zhou Q-Y, Palmiter RD. Dopamine-deficient mice are severely hypoactive, adipsic, and aphagic. Cell. 1995;83(7):1197-209.

60. Berridge KC, Valenstein ES. What psychological process mediates feeding evoked by electrical stimulation of the lateral hypothalamus? Behavioral neuroscience. 1991;105(1):3.

61. Woods SC, Lotter EC, McKay LD, Porte D Jr. Chronic intracerebroventricular infusion of insulin reduces food intake and body weight of baboons. Nature. 1979;282(5738):503.

62. Figlewicz D, Evans S, Murphy J, Hoen M, Baskin D. Expression of receptors for insulin and leptin in the ventral tegmental area/substantia nigra (VTA/SN) of the rat. Brain research. 2003;964(1):107-15.

63. Pardini AW, et al. Distribution of insulin receptor substrate-2 in brain areas involved in energy homeostasis. Brain research. 2006;1112(1):169-78.

64. Figlewicz DP, Szot P, Chavez M, Woods SC, Veith RC. Intraventricular insulin increases dopamine transporter mRNA in rat VTA/substantia nigra. Brain research. 1994;644(2):331-4.

65. Williams JM, et al. Hypoinsulinemia regulates amphetamineinduced reverse transport of dopamine. PLoS biology. 2007;5(10):e274.

66. Carvelli L, et al. PI 3-kinase regulation of dopamine uptake. Journal of neurochemistry. 2002;81(4):859-69.

67. Labouèbe $\mathrm{G}$, et al. Insulin induces long-term depression of ventral tegmental area dopamine neurons via endocannabinoids. Nature neuroscience. 2013;16(3):300.

68. Bruijnzeel AW, Corrie LW, Rogers JA, Yamada H. Effects of insulin and leptin in the ventral tegmental area and arcuate hypothalamic nucleus on food intake and brain reward function in female rats. Behavioural brain research. 2011;219(2):254-64.

69. Mebel DM, Wong JC, Dong YJ, Borgland SL. Insulin in the ventral tegmental area reduces hedonic feeding and suppresses dopamine concentration via increased reuptake. European Journal of Neuroscience. 2012;36(3):2336-46.

70. Figlewicz DP, Bennett J, Evans SB, Kaiyala K, Sipols AJ, Benoit SC. Intraventricular insulin and leptin reverse place preference conditioned with high-fat diet in rats. Behavioral neuroscience. 2004;118(3):479.

71. Dunn JP, et al. Relationship of dopamine type 2 receptor binding potential with fasting neuroendocrine hormones and insulin sensitivity in human obesity. Diabetes care. 2012:DC_112250.

72. Anthony $\mathrm{K}$, et al. Attenuation of insulin-evoked responses in brain networks controlling appetite and reward in insulin resistance: the cerebral basis for impaired control of food intake in metabolic syndrome? Diabetes. 2006;55(11):2986-92. 
73. Volkow ND, et al. "Nonhedonic" food motivation in humans involves dopamine in the dorsal striatum and methylphenidate amplifies this effect. Synapse. 2002;44(3):175-80.

74. Chechlacz M, et al. Diabetes dietary management alters responses to food pictures in brain regions associated with motivation and emotion: a functional magnetic resonance imaging study. Diabetologia. 2009;52(3):524.

75. MacDonald IA. A review of recent evidence relating to sugars, insulin resistance and diabetes. European journal of nutrition. 2016;55(2):17-23.

76. van Golen LW, et al. Effects of insulin detemir and NPH insulin on body weight and appetite-regulating brain regions in human type 1 diabetes: a randomized controlled trial. Plos one. 2014;9(4):e94483.

77. Hermansen K, Fontaine P, Kukolja K, Peterkova V, Leth G, Gall MA. Insulin analogues (insulin detemir and insulin aspart) versus traditional human insulins (NPH insulin and regular human insulin) in basal-bolus therapy for patients with type 1 diabetes. Diabetologia. 2004;47(4):622-9.

78. Jastreboff AM, Sinha R, Lacadie C, Small DM, Sherwin RS, Potenza MN. Neural correlates of stress-and food cue-induced food craving in obesity: association with insulin levels. Diabetes care. 2013;36(2):394-402.

79. Haltia LT, et al. Effects of intravenous glucose on dopaminergic function in the human brain in vivo. Synapse. 2007;61(9):748-56.

80. Vilsbøll T, Krarup T, Madsbad S, Holst JJ. Both GLP-1 and GIP are insulinotropic at basal and postprandial glucose levels and contribute nearly equally to the incretin effect of a meal in healthy subjects. Regulatory peptides. 2003;114(2-3):115-21.

81. Flint A, Raben A, Astrup A, Holst JJ. Glucagon-like peptide 1 promotes satiety and suppresses energy intake in humans. The Journal of clinical investigation. 1998;101(3):515-20.
82. Wang G-J, et al. BMI modulates calorie-dependent dopamine changes in accumbens from glucose intake. PloS one. 2014;9(7): e101585.

83. Burger KS, Stice E. Greater striatopallidal adaptive coding during cue-reward learning and food reward habituation predict future weight gain. Neuroimage. 2014;99:122-8.

84. Stice E, Yokum S, Blum K, Bohon C. Weight gain is associated with reduced striatal response to palatable food. Journal of Neuroscience. 2010;30(39):13105-9.

85.• Ilyas A, et al. The metabolic underpinning of eating disorders: a systematic review and meta-analysis of insulin sensitivity. Molecular and cellular endocrinology. 2018; This paper presents a systematic review and meta-analysis of variations in insulin sensitivity across the spectrum of eating disorders. The metaanalysis supported greater insulin sensitivity in anorexia nervosa and decreased insulin sensitivity in populations with bulimia nervosa and binge eating disorder.

86. Monteleone P, Bortolotti F, Fabrazzo M, La Rocca A, Fuschino A, Maj M. Plasma leptin response to acute fasting and refeeding in untreated women with bulimia nervosa. The Journal of Clinical Endocrinology \& Metabolism. 2000;85(7):2499-503.

87. Frank GK, et al. Altered brain activity in women recovered from bulimic-type eating disorders after a glucose challenge: a pilot study. Int J Eat Disord. 2006;39(1):76-9.

Publisher's Note Springer Nature remains neutral with regard to jurisdictional claims in published maps and institutional affiliations. 